\title{
Correction to: Orius laevigatus strengthens its role as a biological control agent by inducing plant defenses
}

\author{
Sarra Bouagga ${ }^{1}$ - Alberto Urbaneja ${ }^{1} \cdot$ José L. Rambla ${ }^{2} \cdot$ Antonio Granell $^{2} \cdot$ Meritxell Pérez-Hedo $^{1,3}$
}

Published online: 11 March 2021

○) Springer-Verlag GmbH Germany, part of Springer Nature 2021

Correction to: J Pest Sci (2018) 91:55-64

https://doi.org/10.1007/s10340-017-0886-4

The authors regret that values of $\chi^{2}$ and $P$ were published incorrectly. Therefore, the authors would like to include the following changes in the published article:

\section{- In the Key message; where it says}

“..... The results further show how the plant's response to O. laevigatus increases emission of herbivore-induced plant volatiles (HIPVs) which can modulate the behavior of other arthropods (Bemisia tabaci, Frankliniella occidentalis and Encarsia formosa)...", it should say:

“..... The results further show how the plant's response to O. laevigatus increases emission of herbivore-induced plant volatiles (HIPVs) which can modulate the behavior of other arthropods (Bemisia tabaci and Frankliniella occidentalis) ...."

The original article can be found online at https://doi.org/10.1007/ s10340-017-0886-4.

Meritxell Pérez-Hedo

meritxell_p@hotmail.com

1 Centro de Protección Vegetal y BiotecnologíaUnidad de Entomología UJI-IVIA, Instituto Valenciano de Investigaciones Agrarias (IVIA), Carretera de Moncada-Náquera Km. 4,5, 46113 Moncada, Valencia, Spain

2 Instituto de Biología Molecular y Celular de Plantas (IBMCP), Consejo Superior de Investigaciones Científicas, Universidad Politécnica de Valencia, Camino de Vera s/n, 46022 Valencia, Spain

3 Unitat Associada D'Entomologia UJI-IVIA; Departament de Ciències Agràries i del Medi Natural, Universitat Jaume I UJI, Campus del Riu Sec, 12071 Castelló de la Plana, Spain

\section{- In the Abstract; where it says}

“..... These O. laevigatus plant-induced defenses are then shown to contribute to the repellence or attraction of pests or natural enemies, respectively. Specifically, O. laevigatus-punctured sweet pepper plants induce repellency for the whitefly Bemisia tabaci and the thrips species F. occidentalis. In contrast, the whitefly parasitoid Encarsia formosa was significantly attracted to $O$. laevigatus-punctured plants. The plant responses to $O$. laevigatus punctures include the release of an altered blend of volatiles ....", it should say:

“..... These O. laevigatus plant-induced defenses are then shown to contribute to the repellence of pests. Specifically, O. laevigatus-punctured sweet pepper plants induce repellency for the whitefly Bemisia tabaci and the thrips species $F$. occidentalis. The plant responses to $O$. laevigatus punctures include the release of an altered blend of volatiles ...."

- In the Results section; in "Frankliniella occidentalis and $B$. tabaci plant selection mediated by $O$. laevigatus" where it says:

"In the Y-tube experiment, females of $F$. occidentalis showed preference for the odor emitted from intact plants over that from $O$. laevigatus-punctured plants $\left(\chi^{2}=19.931\right.$; $P<0.0001$ ) (Fig. 3). In the case of B. tabaci, tested females were also attracted to the odor emitted by intact sweet pepper plants in comparison to $O$. laevigatus-punctured plants $\left(\chi^{2}=17.071 ; P<0.0001\right)$ (Fig. 3). In contrast to both phytophagous insects, the parasitoid E. formosa significantly chose $O$. laevigatus-punctured plants over intact plants in the olfactometer assay $\left(\chi^{2}=6.250 ; P=0.0124\right)$ (Fig. 3).", it should say:

"In the Y-tube experiment, females of $F$. occidentalis showed preference for the odor emitted from intact plants over that from $O$. laevigatus-punctured plants $\left(\chi^{2}=9.966\right.$; $P=0.0016$ ) (Fig. 3). In the case of B. tabaci, tested females were also attracted to the odor emitted by intact sweet 
pepper plants in comparison to O. laevigatus-punctured plants $\left(\chi^{2}=8.533 ; P=0.0035\right)$ (Fig. 3). In contrast to both phytophagous insects, the parasitoid $E$. formosa did not show a significant preference $\left(\chi^{2}=3.125 ; P=0.0771\right)$ when given a choice between $O$. laevigatus-punctured plants or intact plants in the olfactometer assay (Fig. 3)."

\section{- In the first paragraph of the Discussion; where it says}

"Furthermore, the modulated behavior of both pest species (B. tabaci and F. occidentalis) and a natural enemy ( $E$. formosa) associated with sweet pepper, in response to....", it should say:

"Furthermore, the modulated behaviour of both pest species (B. tabaci and F. occidentalis) associated with sweet pepper, in response to...."

\section{- In the paragraph of the Discussion beginning with "Most of the plant defenses..."; where it says}

"It is shown here that activation of the JA and SA pathways in O. laevigatus-punctured plants is associated with aversion of both arthropod pests, B. tabaci and F. occidentalis, and by contrast, the whitefly parasitoid $E$. formosa was significantly attracted to $O$. laevigatus-punctured plants. Pérez-Hedo et al. (2015a) observed that the ....", it should say:

"It is shown here that activation of the JA and SA pathways in O. laevigatus-punctured plants is associated with aversion of both arthropod pests, B. tabaci and F. occidentalis. Pérez-Hedo et al. (2015a) observed that the...."

\section{- In Discussion, in the first paragraph of the las page;} where it says

"Therefore, the responses of B. tabaci, F. occidentalis and E. formosa were a direct consequence to the odor emitted from the O. laevigatus-punctured sweet pepper plants.", it should say:

"Therefore, the responses of $B$. tabaci and F. occidentalis were a direct consequence to the odor emitted from the $O$. laevigatus-punctured sweet pepper plants."

- In Discussion, in the second paragraph of the las page; where it says

"In this paper, it has been shown that the particular volatile profile emitted by the $O$. laevigatus-punctured plant repelled B. tabaci and F. occidentalis and attracted E. formosa.", it should say:

"In this paper, it has been shown that the particular volatile profile emitted by the $O$. laevigatus-punctured plant repelled B. tabaci and F. occidentalis.",

\section{- In the Fig. 3 caption, where it says:}

"Fig. 3 Response in Y-tube olfactometer of females of F. occidentalis $(\mathrm{n}=33)$, B. tabaci $(\mathrm{n}=33)$ and E. formosa $(\mathrm{n}=34)$ to the odor emitted by intact sweet pepper and by sweet pepper plants previously exposed to O. laevigatus. Significant differences based on a $\chi^{2}$-test are marked using * $(P<0.05)$.", it should say.

"Fig. 3 Response in Y-tube olfactometer of females of F. occidentalis $(\mathrm{n}=33)$, B. tabaci $(\mathrm{n}=33)$ and E. formosa $(\mathrm{n}=34)$ to the odor emitted by intact sweet pepper and by sweet pepper plants previously exposed to $O$. laevigatus. "n" indicates the number of individuals tested, "nc" indicates the number of individuals that did not make a choice and "n.s." indicates non-significance. Significant differences based on a $\chi^{2}$-test are marked using $*(P<0.05)$.

- From the new corrected statistical values, Fig. 3 should read: 
O. laevigatus punctured plants

O. laevigatus punctured plants

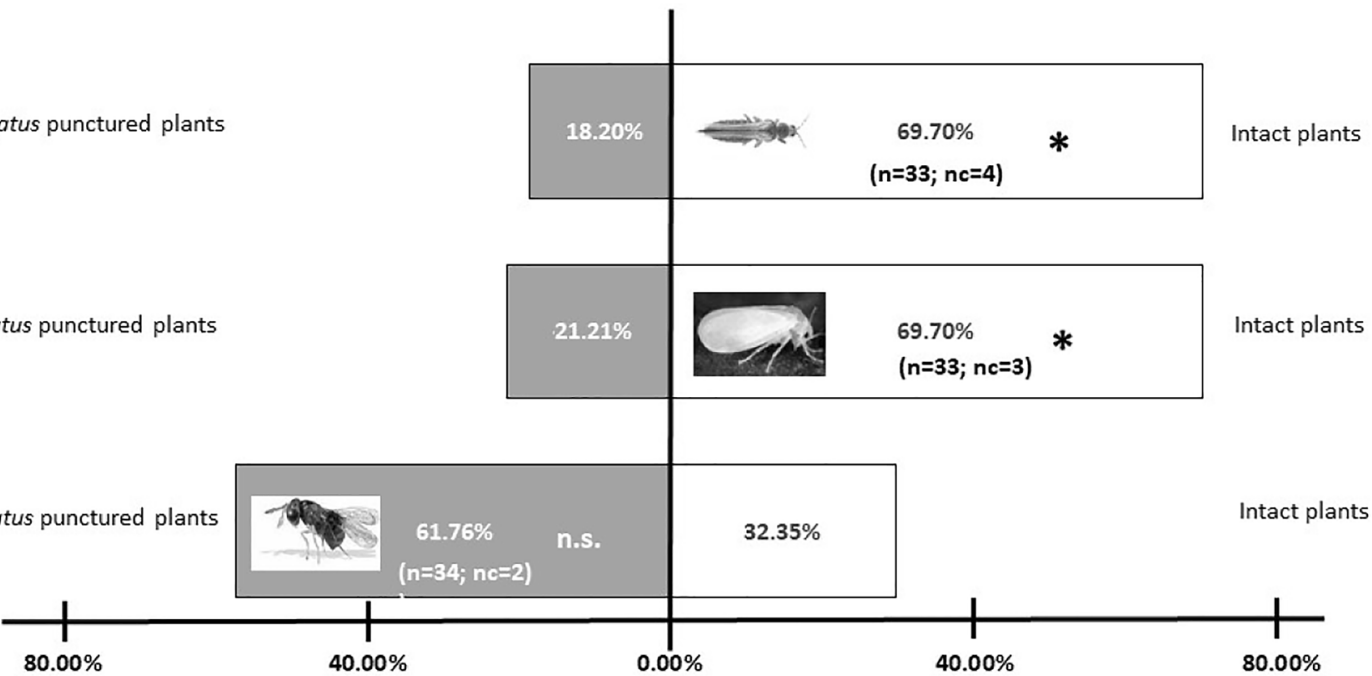

O. laevigatus punctured plants

Publisher's Note Springer Nature remains neutral with regard to jurisdictional claims in published maps and institutional affiliations. 